\title{
Suppression of HIF2 signalling attenuates the initiation of hypoxia-induced pulmonary hypertension
}

\author{
Cheng-Jun $\mathrm{Hu}^{1,2,5,6}$, Jens M. Poth ${ }^{2,3,5}$, Hui Zhang ${ }^{2}$, Amanda Flockton², \\ Aya Laux ${ }^{1}$, Sushil Kumar ${ }^{2}$, Brittany McKeon ${ }^{2}$, Gary Mouradian ${ }^{2}, \mathrm{Min}_{\mathrm{Li}}{ }^{2}$, \\ Suzette Riddle ${ }^{2}$, Steven C. Pugliese ${ }^{2}$, R. Dale Brown², Eli M. Wallace ${ }^{4}$, \\ Brian B. Graham (1) ${ }^{2}$, Maria G. Frid ${ }^{2}$ and Kurt R. Stenmark ${ }^{2,6}$
}

Affiliations: 'Dept of Craniofacial Biology, School of Dental Medicine, University of Colorado, Aurora, CO, USA. ${ }^{2}$ Cardiovascular Pulmonary Research Laboratories, Division of Pulmonary Sciences and Critical Care Medicine, Division of Pediatrics-Critical Care, Depts of Medicine and Pediatrics, University of Colorado, Aurora, CO, USA. ${ }^{3}$ Dept of Anesthesiology and Intensive Care Medicine, University Medical Center, Rheinische Friedrich Wilhelms University of Bonn, Bonn, Germany. ${ }^{4}$ Peloton Therapeutics Inc., Dallas, TX, USA. ${ }^{5}$ These authors share first authorship. ${ }^{6}$ These authors are joint corresponding authors.

Correspondence: Kurt R. Stenmark, Cardiovascular Pulmonary Research Laboratories, Dept of Medicine and Pediatrics, University of Colorado Anschutz Medical Campus, Campus Box B131, 12700 E. 19th Avenue, RC2, Aurora, C0 80045, USA. E-mail: kurt.stenmark@ucdenver.edu

@ERSpublications

Activation of HIF2 by hypoxia initiates vascular cell proliferation and recruitment of inflammatory cells at early stages of PH development through HIF2-dependent transcription of genes involved in these pathways in pulmonary vascular cells http://bit.ly/2lFwTGM

Cite this article as: $\mathrm{Hu}$ C-J, Poth JM, Zhang H, et al. Suppression of HIF2 signalling attenuates the initiation of hypoxia-induced pulmonary hypertension. Eur Respir J 2019; 54: 1900378 [https://doi.org/ 10.1183/13993003.00378-2019].

ABSTRACT Most published studies addressing the role of hypoxia inducible factors (HIFs) in hypoxiainduced pulmonary hypertension development employ models that may not recapitulate the clinical setting, including the use of animals with pre-existing lung/vascular defects secondary to embryonic HIF ablation or activation. Furthermore, critical questions including how and when HIF signalling contributes to hypoxia-induced pulmonary hypertension remain unanswered.

Normal adult rodents in which global HIF1 or HIF2 was inhibited by inducible gene deletion or pharmacological inhibition (antisense oligonucleotides (ASO) and small molecule inhibitors) were exposed to short-term (4 days) or chronic (4-5 weeks) hypoxia. Haemodynamic studies were performed, the animals euthanised, and lungs and hearts obtained for pathological and transcriptomic analysis. Cell-typespecific HIF signals for pulmonary hypertension initiation were determined in normal pulmonary vascular cells in vitro and in mice (using cell-type-specific HIF deletion).

Global Hifla deletion in mice did not prevent hypoxia-induced pulmonary hypertension at 5 weeks. Mice with global Hif2a deletion did not survive long-term hypoxia. Partial Hif2a deletion or Hif2-ASO (but not Hif1-ASO) reduced vessel muscularisation, increases in pulmonary arterial pressures and right ventricular hypertrophy in mice exposed to 4-5 weeks of hypoxia. A small molecule HIF2 inhibitor (PT2567) significantly attenuated early events (monocyte recruitment and vascular cell proliferation) in rats exposed to 4 days of hypoxia, as well as vessel muscularisation, tenascin $\mathrm{C}$ accumulation and pulmonary hypertension development in rats exposed to 5 weeks of hypoxia. In vitro, HIF2 induced a distinct set of genes in normal human pulmonary vascular endothelial cells, mediating inflammation and proliferation of endothelial cells and smooth muscle cells. Endothelial Hif2a knockout prevented hypoxia-induced pulmonary hypertension in mice.

Inhibition of HIF2 (but not HIF1) can provide a therapeutic approach to prevent the development of hypoxia-induced pulmonary hypertension. Future studies are needed to investigate the role of HIFs in pulmonary hypertension progression and reversal. 


\section{Introduction}

Pulmonary hypertension due to lung disease and/or hypoxia (group 3 pulmonary hypertension) affects nearly 1 million people worldwide, making it the second largest group of patients suffering from this disease. Unfortunately, none of the currently approved drugs for group 1 pulmonary arterial hypertension have been shown in randomised controlled trials to benefit patients with group 3 pulmonary hypertension, emphasising the need for a better understanding of disease mechanisms as they might aid in the discovery of new therapies.

There is abundant evidence supporting the central involvement of hypoxia inducible factors (HIFs) in chronic hypoxia-induced pulmonary hypertension [1-11]. However, we believe the experimental approaches used in most previously published studies may not recapitulate the clinical settings. For example, some group 3 pulmonary hypertension development is due to hypoxia exposure in post-natal humans and animals with normal pulmonary circulation. It is important to note that except for two studies in which Hif1a deletion in smooth muscle cells (SMCs) or endothelial cells (ECs) was initiated in adult mice [10, 11], in all other studies Hif1a or Hif2a deletion was initiated in developing embryos [1-9]. It has been well established that all these HIF knockout or activation models (prolyl-4-hydroxylase domain 2 (Phd2) knockout) exhibit vascular defects in developing embryos and likely in adult mice derived from these embryos [12-17]. Also, due to the essential role of HIF in development, most published studies use cell-type-specific Hifa knockout models [2-7, 10, 11]. We believe such cell-type-specific Hifa knockout approaches may have shortcomings for the purpose of determining the general function of Hifa genes in a disease such as pulmonary hypertension where interactions between multiple cell types (ECs, SMCs and fibroblasts) are clearly necessary for disease progression and a specific gene may have different or opposite functions in different cell types involved in the disease.

Besides inducible deletion, pharmacological inhibition could be an important tool to study the general role of HIF in pulmonary hypertension development in normal adult animals. Given the potential important role of HIF proteins and particularly HIF2 in pulmonary hypertension, successful pharmacological inhibition could also lay an important foundation for potential pulmonary hypertension treatment. Recent studies have led to small molecules that specifically block HIF2 (but not HIF1) activity [18, 19]. These inhibitors have also been shown to exhibit strong antitumor activity in vivo and in vitro [18, 20]. Additionally, antisense oligonucleotides (ASOs) to HIF1 and HIF2 have also been developed, and shown to attenuate HIF expression in vivo and to abrogate HIF-mediated disease pathology [21].

In the study presented here, we sought to re-evaluate the role of HIFs in hypoxia-induced pulmonary hypertension using approaches that may better recapitulate the clinical settings. Specifically, HIF activity is inhibited by inducible gene deletion and pharmacological inhibitors in which global (not cell-type-specific) HIF1 or HIF2 activity inhibition is initiated in normal adult animals without any predisposed diseases resulting from embryonic deletion of HIFs. In addition, we sought to better understand the underlying molecular mechanisms concerning how HIFs promote pulmonary hypertension development, by studying the role of HIF in early as well as late stages of pulmonary hypertension development.

\section{Methods}

See the supplementary material for full details.

\section{Statistics}

In general, data is reported as mean \pm SEM. Statistical differences were evaluated by two-way ANOVA as well as the unpaired two-tailed t-test. Figure legends specify the statistical analysis used for the data in each panel. $\mathrm{p}<0.05$ was considered statistically significant. Group size (n) is reported in the corresponding figure legends. Data were logged using Excel (Microsoft, Redmond, WA, USA), graphing and calculations were performed using Prism (GraphPad, La Jolla, CA, USA), and figures were designed using PowerPoint (Microsoft).

\section{Results}

HIF1 $\alpha$ is dispensable in establishing hypoxia-induced pulmonary hypertension at 5 weeks in adult mice

To delete the Hifla gene in adult mice globally, Hif1 $a^{f l / f l}$; $\mathrm{UbcCreERT}^{+}$and Hifl $a^{f l f l}$; $\mathrm{UbcCreERT}^{-}$(control) mice were injected with tamoxifen for 5 days (see the supplementary material for full details of transgenic mice and genotyping). Mice were then allowed to rest for 1 week before exposure to either hypobaric

This article has supplementary material available from erj.ersjournals.com

Received: 22 Feb 2019 | Accepted after revision: 28 Aug 2019 
hypoxia $\left(P_{\mathrm{B}}=370 \mathrm{mmHg}\right.$; simulated altitude of $\left.\sim 18000 \mathrm{ft} \quad(\sim 5500 \mathrm{~m})\right)$ or normobaric normoxia $\left(P_{\mathrm{B}}=740 \mathrm{mmHg}\right.$ ) for 5 weeks (figure 1a). After 5 weeks of hypoxia exposure, readout parameters were measured and animals were euthanised. The efficiency of Hifla deletion in UbcCreERT ${ }^{+}$mice under hypoxic conditions is $80 \%$ (supplementary table S1). Hypoxia exposure induced increases of haematocrit (Hct) (figure 1b), right ventricular systolic pressure (RVSP) (figure 1c) and right ventricular hypertrophy, as indicated by increases in the Fulton index (ratio of the weight of the right ventricle to the weight of the left ventricle and septum) (figure 1d) and in the ratio of the weight of the right ventricle to body weight $\left(\right.$ weight $_{\mathrm{RV}} /$ weight $_{\text {Body }}$ ) (figure $1 \mathrm{e}$ ), in both $\mathrm{Hifl}^{\text {fllfl }}$; $\mathrm{UbcCreERT}^{-}$and Hifl $a^{\text {flffl }}$; $\mathrm{UbcCreERT}^{+}$mice. Hypoxia also similarly reduced body weight and increased heart rate in both UbcCreERT ${ }^{+}$and UbcCreERT $^{-}$ animals compared with normoxic animals (supplementary table S2). Thus, our data demonstrate that Hifla expression is dispensable for development of pulmonary hypertension and right ventricle hypertrophy in normal adult mice after 5 weeks of hypoxia.

\begin{tabular}{|c|c|c|c|c|c|c|c|}
\hline Time & Week 1 & Week 2 & Week 3 & Week 4 & Week 5 & Week 6 & Week 7 \\
\hline \multirow{2}{*}{ Pressure } & \multirow{2}{*}{ Ambient } & \multirow{2}{*}{ Sea level } & $18000 \mathrm{ft}$ & $18000 \mathrm{ft}$ & $18000 \mathrm{ft}$ & $18000 \mathrm{ft}$ & $18000 \mathrm{ft}$ \\
\hline & & & Sea level & Sea level & Sea level & Sea level & Sea level \\
\hline
\end{tabular}

$\operatorname{TMX}+-$
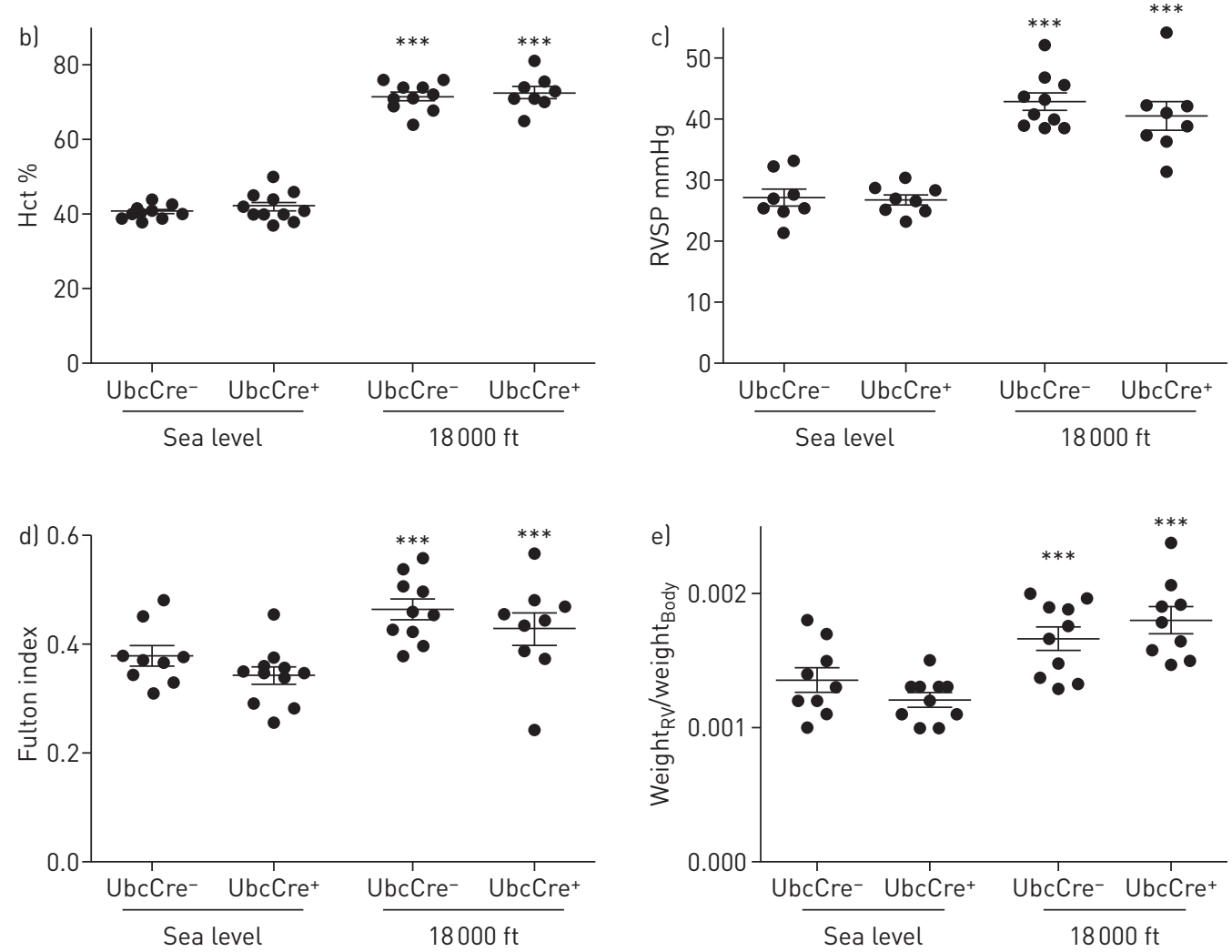

FIGURE 1 Global hypoxia inducible factor $1 \alpha$ (Hif1a) is dispensable in establishing hypoxia-induced pulmonary hypertension at 5 weeks in adult mice. TMX: tamoxifen; Hct: haematocrit; UbcCre: fusion protein consisting of Cre recombinase and the human oestrogen receptor binding domain under control of a ubiquitously active ubiquitin $\mathrm{C}$ gene promoter, in which Cre recombinase is activated upon TMX exposure; RVSP: right ventricular systolic pressure. a) Experimental setup: TMX was injected into mice daily during week 1 to activate Cre and delete Hif1a, then all mice were moved to sea level for 1 week, followed by exposing mice to either normoxia $\left(P_{\mathrm{B}}=740 \mathrm{mmHg}\right)$ or hypoxia (simulated altitude of $\left.\sim 18000 \mathrm{ft}(\sim 5500 \mathrm{~m}) ; P_{\mathrm{B}}=370 \mathrm{mmHg}\right)$ for 5 weeks. After 5 weeks of hypoxia exposure, readout parameters were measured and animals were euthanised. b) Hct levels, c) RVSP, d) Fulton index and e) ratio of the weight of the right ventricle to bodyweight (weight RV $/$ weight $_{\text {Body }}$ ). Animal numbers in each group under normoxia or hypoxia can be found in the Fulton index panel (d). Due to technical difficulties, we were not able to obtain readings of Hct and/or RVSP for some mice, thus the animal numbers for these data were typically less than the animal numbers for the Fulton index in this and other figures of this study. ${ }^{* *}: p<0.001$, difference between hypoxia versus normoxia in the same genotype lor treatment) group. Statistical analysis by two-way ANOVA. 
Mice with global genetic deletion of HIF2 $\alpha$ do not survive long-term hypoxia

To investigate the role of Hif $2 a$ in hypoxia-induced pulmonary hypertension development in normal adult mice, adult $H$ if $2 a^{f l / f l}$; $\mathrm{UbcCreERT}^{+}$and $\mathrm{Hif} 2 a^{f l f f l}$; $\mathrm{UbcCreERT}^{-}$mice were similarly treated with tamoxifen and exposed to normoxia or hypoxia, as we did for Hifla mice. The efficiency of Hif2a deletion in hypoxic Hif $2 a^{f l / f l}$; $\mathrm{UbcCreERT}^{+}$mice at the end of the experiment was $71 \%$ (supplementary table S1). While there were no differences in survival and pulmonary hypertension development among Hif $2 a^{f l / f l}$; $\mathrm{UbcCreERT}^{-}$,

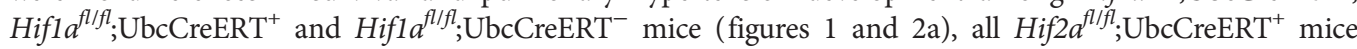
died within 4 weeks of hypoxia exposure (figure 2a). These data indicate that global Hif2a (but not Hifla) deletion is not compatible with survival of mice exposed to chronic hypoxic conditions.

\section{Global partial HIF2 $\alpha$ gene deletion diminishes development of hypoxia-induced pulmonary} hypertension at 5 weeks in adult mice

To prevent lethality, we generated $H$ if $2 a^{\text {wt } / f l} ; \mathrm{UbcCreERT}^{+}$and $H$ Hif $2 a^{\text {wt } / f l}$; $\mathrm{UbcCreERT}^{-}$mice, and then treated them with tamoxifen and exposed them to hypoxia for 5 weeks. The efficiency of Hif $2 a$ deletion in hypoxic Hif $2 a^{w t / f l}$; $\mathrm{UbcCreERT}^{+}$mice at the end of the experiment was $36 \%$ (supplementary table S1). Cre-negative animals displayed increases in Hct (figure 2b), RVSP (figure 2c), Fulton index (figure 2d) and weight $_{\mathrm{RV}} /$ weight $_{\mathrm{Body}}$ ratio (figure $2 \mathrm{e}$ ) in response to hypoxia. While there were still significant increases in these parameters in hypoxia-exposed Hif $2 a^{\text {wt } / f l}$; UbcCreERT ${ }^{+}$mice, the level of increase for some parameters was significantly reduced in comparison with Cre-negative littermates (figure $2 \mathrm{~d}$ and e). Thus, our data, generated from normal adult mice, demonstrate that reduction of Hif $2 a$ attenuates development of pulmonary hypertension induced by 5 weeks of hypoxia exposure.

\section{Knockdown of Hif2a utilising ASOs significantly reduces development of hypoxia-induced pulmonary hypertension at 5 weeks in adult mice}

To further investigate the role of Hif $2 a$ in hypoxia-induced pulmonary hypertension in normal adult mice and to examine whether targeting Hif2a therapeutically in adult mice under hypoxic conditions would be beneficial or lead to lethality, we used an ASO approach. The effectiveness and feasibility of Hif2a-ASO in reducing Hif $2 a$ expression in adult mice maintained under normoxia was tested in a pilot experiment, which showed an up to $90 \%$ reduction of Hif 2 a mRNA levels in lungs, spleens, kidneys and livers in mice treated with Hif2a-ASO two times per week for 2 weeks (figure 3a, left), without significant changes of Hifla mRNA in all organs examined (figure 3a, right). Furthermore, Hif2a-ASO also reduced expression of the HIF2 target gene Epo1, but not the HIF1 target gene Pgk1, in kidneys (figure $3 \mathrm{~b}$ ).

To determine the general role of HIF in hypoxia-induced pulmonary hypertension development, mice were injected with Hif2a-ASO or a control-ASO (unspecific, no known target) for 1 week with two injections per week and then exposed to either normoxia or hypoxia for 5 weeks during which two injections of ASO per week were maintained (figure 3c). The efficiency of Hif2a deletion in hypoxic mice targeted with Hif $2 a$-ASO was $72 \%$ at the end of the experiment (supplementary table S1). We did not observe any lethality in Hif2a-ASO-treated mice under hypoxia. However, hypoxic Hif2a-ASO-treated mice exhibited a trend towards increased weight loss (supplementary table S4). Further studies also revealed that levels of circulating catecholamines (epinephrine and norepinephrine) were reduced by Hif2a-ASO treatment, as were heart rate, cardiac output and maximal pressure increase $\mathrm{d} P / \mathrm{d} t_{\max }$ (supplementary figure S2b-e). In addition, two animals died during readout procedures. Taken together, these findings suggest an increased fragility in these mice.

Hct in Hif2a-ASO-treated mice was lower, both under normoxia and under hypoxia (figure 3d). Under hypoxic conditions, Hif2a-ASO-treated mice also exhibited a reduction in RVSP (figure 3e), Fulton index (figure $3 \mathrm{f}$ ) and weight $\mathrm{RV}_{\mathrm{RV}}$ /weight $\mathrm{Body}_{\mathrm{B}}$ ratio (figure $3 \mathrm{~g}$ ) compared with hypoxic control-ASO mice. Consistent with haemodynamic data, hypoxia-exposed Hif2a-ASO-treated animals exhibited a marked reduction in fully muscularised vessels in the lungs compared with hypoxia-exposed control-ASO mice (figure $3 \mathrm{~h}-\mathrm{j}$ ).

However, Hifla-ASO reduced Hif1a mRNA by $60 \%$ in lung tissues (supplementary table S1), but it had no effect on Hct, RVSP or the Fulton index in hypoxic mice (figure 3k), similar to the lack of effect observed in inducible Hif1a knockout mice (figure 1).

\section{A small molecule HIF2 inhibitor PT2567 significantly reduces development of hypoxia-induced pulmonary hypertension at 4 weeks in adult rats}

To further address the safety of HIF2 inhibition and the role of HIF2 in initiation of hypoxia-induced pulmonary hypertension, we treated rats (which develop more severe pulmonary hypertension than mice) with a small molecule inhibitor (PT2567; Peloton Therapeutics, Dallas, TX, USA) that specifically blocks HIF2 activity $[18,19]$. Exposure to 4 weeks of hypoxia led to pulmonary hypertension development in rats treated with the control reagent $(0.5 \%$ methylcellulose $/ 0.5 \%$ Tween- 80$)$, as demonstrated by increased 

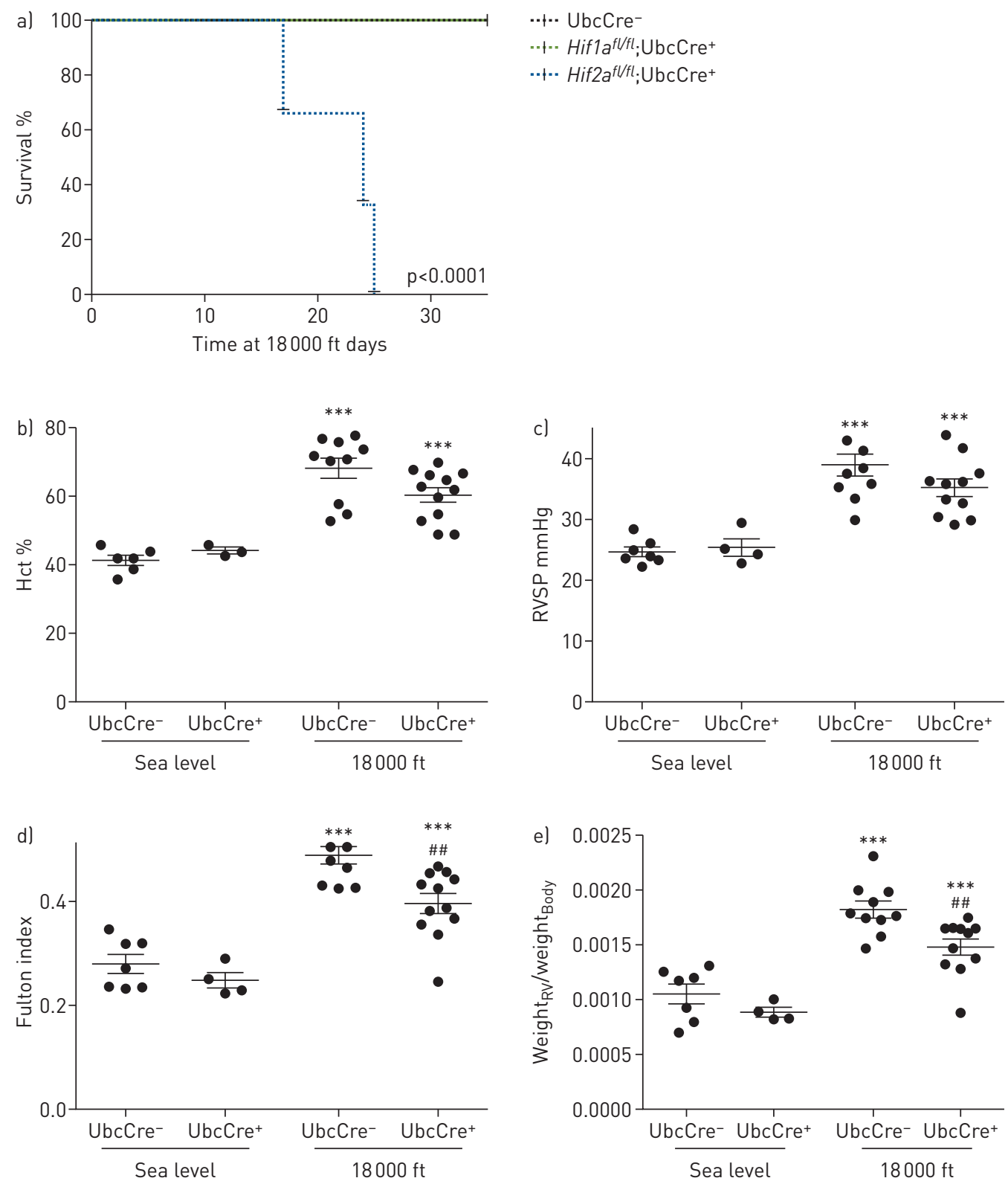

FIGURE 2 Global Hif2a deletion is incompatible for mouse survival under hypoxic conditions, while global partial Hif2a deletion diminishes hypoxia-induced pulmonary hypertension development at 5 weeks in adult mice. UbcCre: fusion protein consisting of Cre recombinase and the human oestrogen receptor binding domain under control of a ubiquitously active ubiquitin $C$ gene promoter, in which Cre recombinase is activated upon tamoxifen exposure; Hct: haematocrit; RVSP: right ventricular systolic. Mice were treated with tamoxifen and exposed to normoxia or hypoxia as described in figure 1a. a) Kaplan-Meier curve for survival of Hif2 $2 a^{f l / f l}$ :UbcCreERT ${ }^{+}$mice during exposure to hypoxia compared with survival of Hifla $a^{f l / f l} ;$ UbcCreERT $^{+}$and Hif2a ${ }^{f l / f l}$;UbcCreERT ${ }^{-}$mice during exposure to hypoxia. $n>9$ mice in each group were used for this experiment. b-e) Haemodynamics of Hif2a $a^{f / w t}$;UbcCreERT ${ }^{+}$and Hif2a $a^{f / / w t}$;UbcCreERT ${ }^{-}$mice after 5 weeks of exposure to normoxia or hypoxia: b) Hct, c) RVSP, d) Fulton index and e) ratio of the weight of the right ventricle to body weight (weight $\mathrm{RV}_{\mathrm{R}} /$ weight $_{\mathrm{Body}}$ ). ${ }^{* * *}: \mathrm{p}<0.001$, difference between hypoxia versus normoxia in the same genotype lor treatment) group; ${ }^{\# \#}: p<0.01$, difference between genotypes or treatments under hypoxic conditions. Statistical analysis shown here is two-way ANOVA analysis (note in (c) there is a significant decrease in RVSP under hypoxia in $\mathrm{UbcCre}^{+}$animals analysed by the unpaired t-test: $\left.\mathrm{p}<0.05\right)$.

mean pulmonary arterial pressure (mPAP) (figure 4a) and increased Fulton index (figure 4b). The HIF2 inhibitor PT2567 reduced mPAP (figure 4a) and attenuated right ventricle remodelling (figure 4b). To better understand the molecular mechanisms underlying HIF2's role in pulmonary hypertension development in adult animals, we examined multiple pathways and genes that are important in pulmonary hypertension development [3-7, 22-24]. Indeed, increased inflammatory cell accumulation (monocytes) 

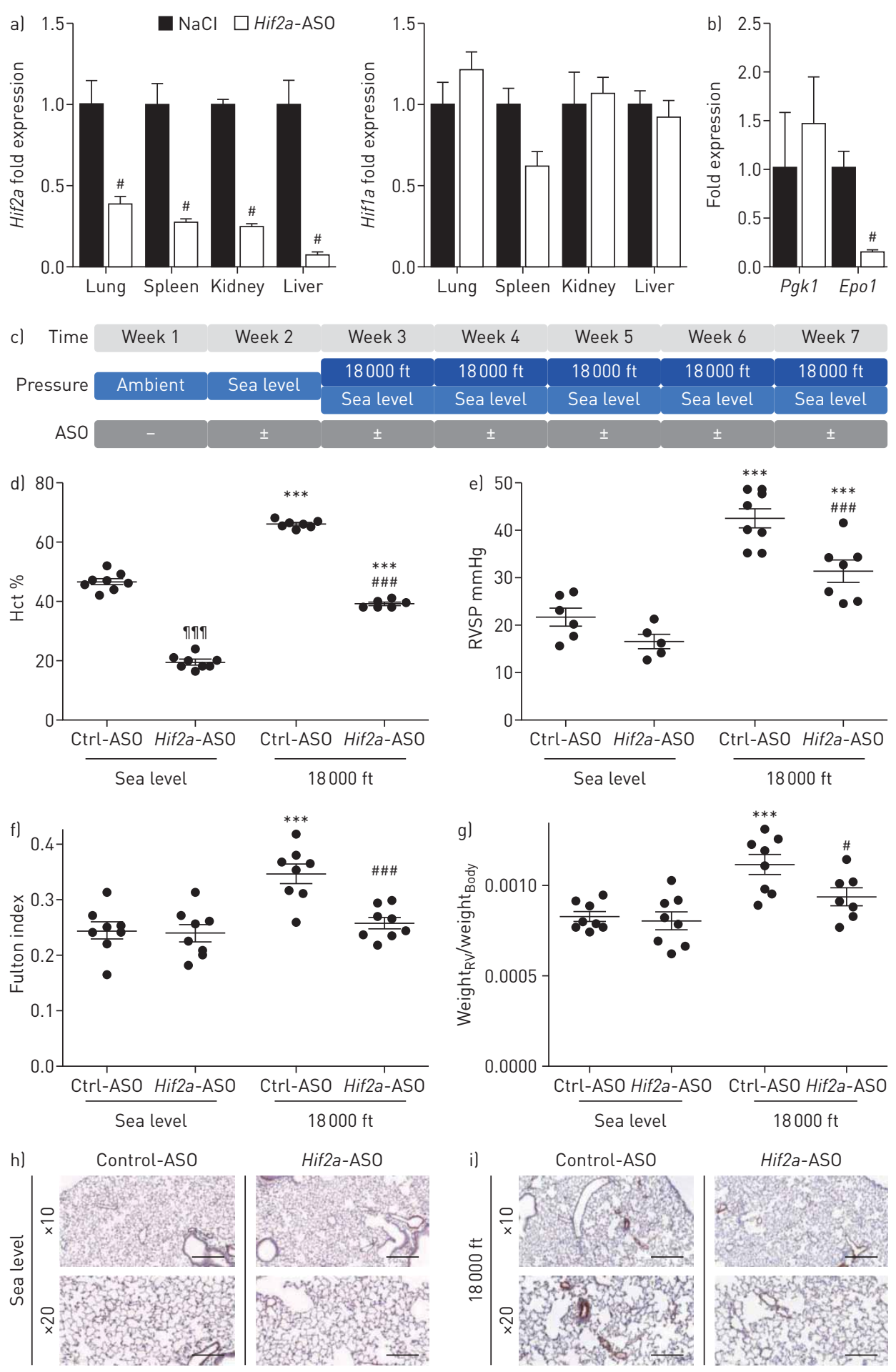

Hif2a-ASO

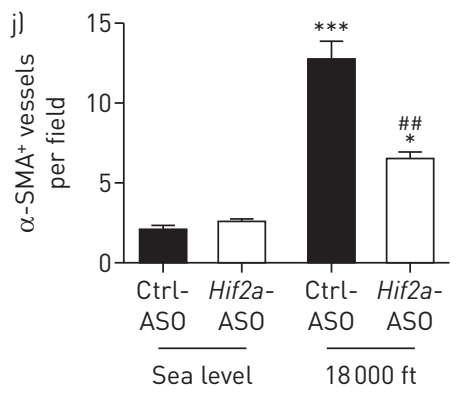

k)

\begin{tabular}{lccc}
\hline & Control-ASO & Hif1a-ASO & p-value \\
\hline HCT \% & $66 \pm 0.5$ & $67.9 \pm 2.17$ & 0.1412 \\
RVSP mmHg & $42.62 \pm 2.17$ & $43.08 \pm 1.60$ & 0.8608 \\
Fulton index & $0.3455 \pm 0.0185$ & $0.3457 \pm 0.0173$ & 0.9940 \\
Weight $_{\text {RV }} /$ weight $_{\text {Body }}$ & $0.00112 \pm 0.00006$ & $0.00110 \pm 0.00007$ & 0.8656
\end{tabular}


FIGURE 3 Knockdown of Hif2a but not Hifla utilising antisense oligonucleotides (ASOs) significantly reduces development of hypoxia-induced pulmonary hypertension at 5 weeks in adult mice. Hct: haematocrit; RVSP: right ventricular systolic; $\alpha$-SMA: $\alpha$-smooth muscle actin. a, b) Testing the effectiveness and specificity of ASOs in a pilot experiment. Wild-type C57BL/6 J mice were treated either with injections of an ASO targeting Hif2a mRNA (Hif2a-ASO) or equal volumes of $0.9 \% \mathrm{NaCl}$ (control) at days 1, 4, 8 and 11. At day 12, mice $(\mathrm{n}=3)$ were sacrificed and multiple organs were collected for RNA preparation. a) Levels of Hif1a and Hif2a mRNA were quantified by quantitative real-time PCR in the indicated organs. b) Levels of Pgk1 (a HIF1 target gene) and Epo1 (a HIF2 target gene) in kidneys from mice targeted with $\mathrm{NaCl}$ or Hif2a-ASO were quantified by quantitative real-time PCR. c) Experimental setup. In week 2, mice were kept at sea level and began to receive injections of control (ctrl), Hifla-ASO or Hif2a-ASO (two injections per week on Monday and Thursday). Starting week 3, mice were exposed to either normoxia (sea level) or hypoxia (simulated altitude of $\sim 18000 \mathrm{ft}$ $(\sim 5500 \mathrm{~m}))$ for 5 weeks, at which two injections per week were maintained. $d-k)$ End-point measurements for the experimental animals in (c): d) Hct, e) RVSP, f) Fulton index and g) ratio of the weight of the right ventricle to body weight ( weight $_{\mathrm{RV}} /$ weight $_{\text {Body }}$ ). $\mathrm{h}$-j) $\alpha$-SMA-positive pulmonary vessels. Scale bar: $200 \mu \mathrm{m}$ (top row); $100 \mu \mathrm{m}$ (bottom row). k) Summary of Hct, RVSP, Fulton index and weight $_{\text {RV }} /$ weight $_{\text {Body }}$ for mice targeted with control-ASO or Hif1a-ASO under normoxia or hypoxia. ${ }^{*}: p<0.05 ;{ }^{* * *}: p<0.001$, difference between hypoxia versus normoxia in the same genotype (or treatment) group; ${ }^{\#}: p<0.05 ;{ }^{\# \#}: p<0.01$; ${ }^{\# \# \#: ~} p<0.001$, difference between genotypes or treatments under hypoxic conditions; ๆศा : $p<0.001$, difference between genotypes or treatments under normoxic conditions. Statistical significance as determined by the t-test $(a, b, j$ and $k)$ or two-way ANOVA (d-g).

(figure 4c, top panel), slightly increased numbers of proliferating cells (Ki-67) (figure 4c, middle panel) and increased tenascin C (TNC) expression (figure 4c, lower panel) were observed in hypoxia-exposed rats, which were reduced by the HIF2 inhibitor PT2567 in adventitial areas of pulmonary vessels from hypoxia-exposed rats. The changes in proliferation and TNC expression in hypoxic rats and rats treated with HIF2 inhibitor using immunostaining were confirmed by Ccna1 (cyclin A1, a marker for cell proliferation) and Tnc mRNA levels in lung tissues of the corresponding rats (figure 4c, middle and bottom right). However, the increased monocyte accumulation in hypoxic rats using immunostaining was not confirmed by their mRNA levels in lung tissues of the corresponding rats (figure $4 \mathrm{c}$, top right), likely due to the possibility that macrophages/monocytes are attracted to pulmonary vessels, but the total cell numbers are not significantly altered in the whole lungs of rats exposed to hypoxia for 4 weeks. In addition, more muscularised vessels were observed in hypoxia-exposed rats, which were reduced by the HIF2 inhibitor PT2567 (figure 4d and e), data consistent with our Hif2a-ASO observations in mice. Furthermore, consistent with previous reports [3-7, 23], a number of functionally important genes in pulmonary hypertension such as Icam1, Sdf1, Arg1, Arg2, Ccnd1, Edn1, Pai1, Tgfa and Tsp1 exhibited increased levels in lungs of hypoxia-exposed rats (supplementary figure S3). The increase in the aforementioned genes, but not genes such as Adm, Glut1 and Ndrg1, was significantly reduced in hypoxia-exposed rats treated with the HIF2 inhibitor PT2567 (supplementary figure S3), suggesting that the first group of genes likely represents target (direct or indirect) genes unique to HIF2, while the HIF2 inhibitor-insensitive genes are likely to be regulated in a HIF2-independent manner at this time-point. These data indicate that HIF2 inhibition significantly reduces development of hypoxia-induced pulmonary hypertension, by preventing induction of genes involved inflammation (supplementary figure S3b) and cell signalling (proliferation and fibrotic responses) (supplementary figure S3c).

\section{HIF2 activity is required for increased accumulation of monocytes and increased cell proliferation observed in hypoxia-exposed adult rats at 4 days}

Using both genetic and pharmacological approaches, our aforementioned experiments demonstrated that inhibition of HIF2 is able to attenuate the development of pulmonary hypertension at $4-5$ weeks of hypoxia using normal adult mice and rats as models. Subsequent experiments are intended to address multiple critical remaining questions concerning how HIF2 promotes pulmonary hypertension initiation, but not to determine the general role of HIF2 in hypoxia-induced pulmonary hypertension. The first question we wanted to answer is at which stage of pulmonary hypertension development does HIF2 signalling have an impact? To help address this question, we performed short-term hypoxia experiments. Exposure to 4 days of hypoxia led to moderate increases of mPAP (figure 5a) and the Fulton index (figure $5 b$ ) in rats treated with control reagent, in which only the Fulton index was reduced by the HIF2 inhibitor PT2567 (figure 5b). Consistent with our previous findings $[25,26]$, in short-term hypoxia-exposed rats, changes in TNC expression were minimal (figure 5c), but there was a significant increase in accumulation of monocytes and cell proliferation (figure 5c). The HIF2 inhibitor PT2567 completely abolished these changes (figure 5c). The changes of Cd68, Ccnal and Tnc expression in hypoxic rats and rats treated with HIF2 inhibitor examined by immunostaining were confirmed by their mRNA levels in lungs of the corresponding rats (figure $5 c$, right side). Examination of gene expression in lungs demonstrated that most HIF2-regulated genes (Icam1, Sdf1, Arg1, Arg2, Ccnd1, Edn1 and Pai1, but not Tgfa and Tsp1) that were observed in rats exposed to 4 weeks of hypoxia (supplementary figure S3) also exhibited induction in rats 

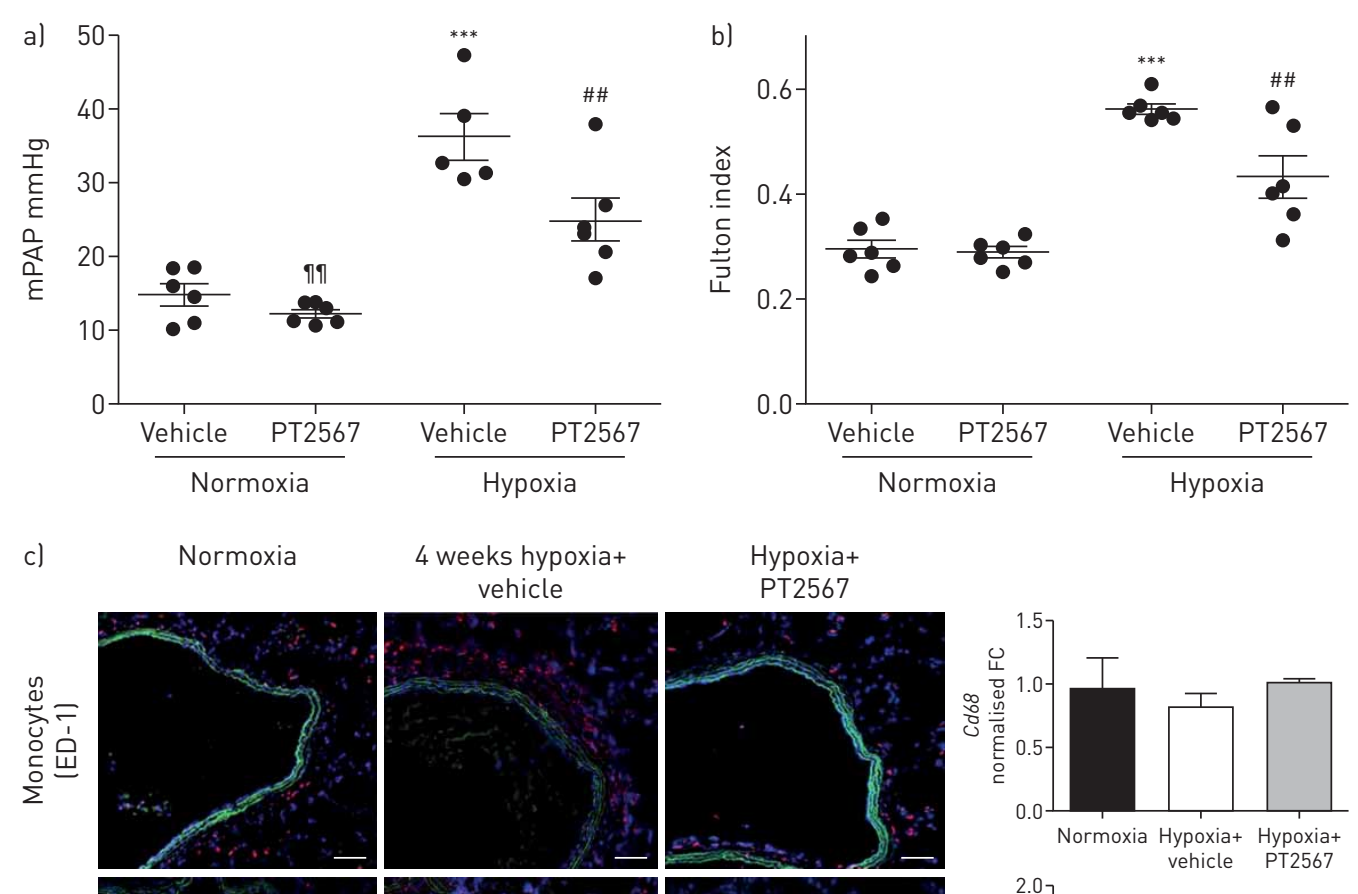

4 weeks hypoxia+ vehicle

Hypoxia+ PT2567
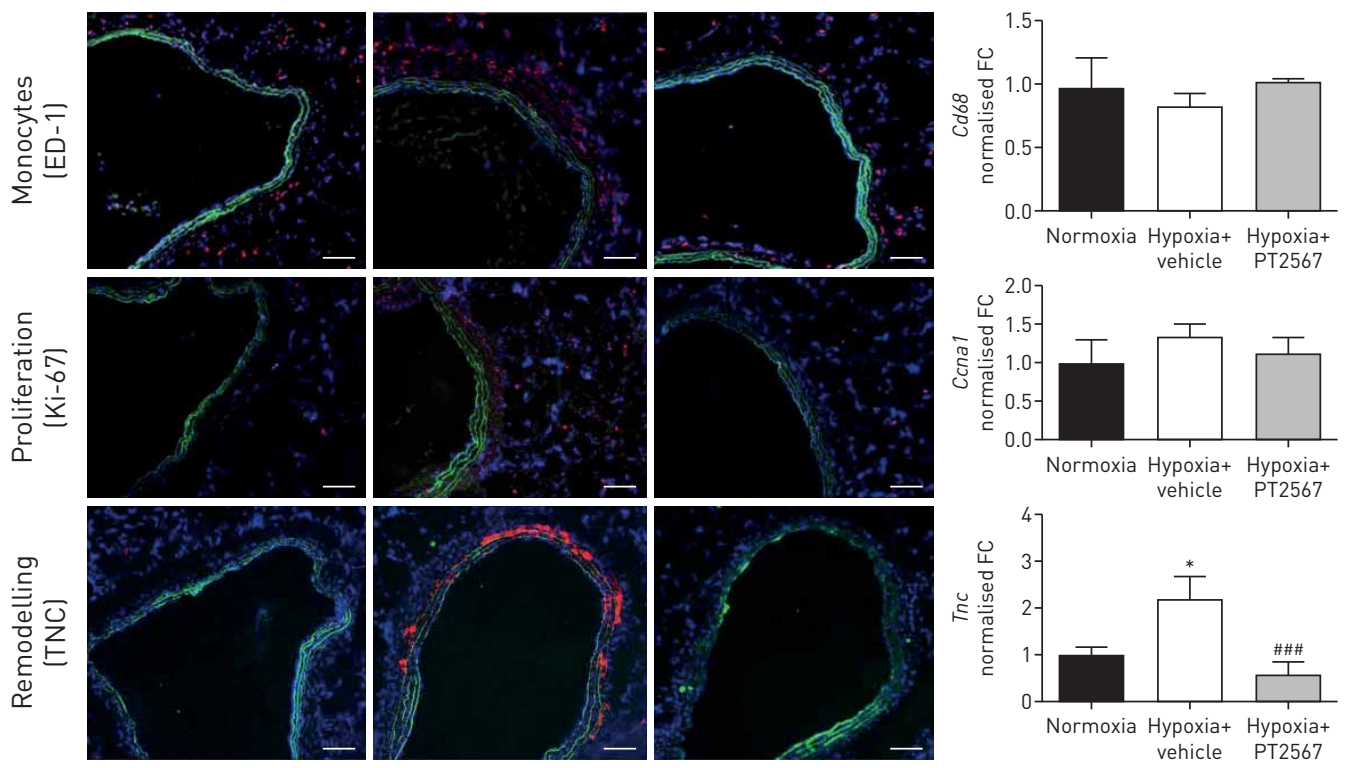

d) Normoxia+ vehicle

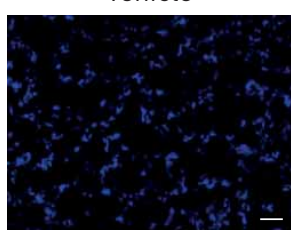

4 weeks hypoxia+ vehicle

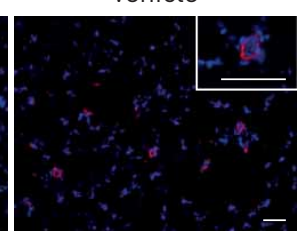

Hypoxia+ PT2567

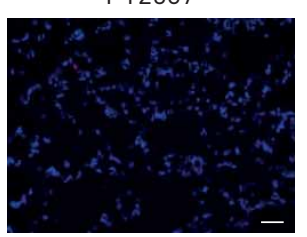

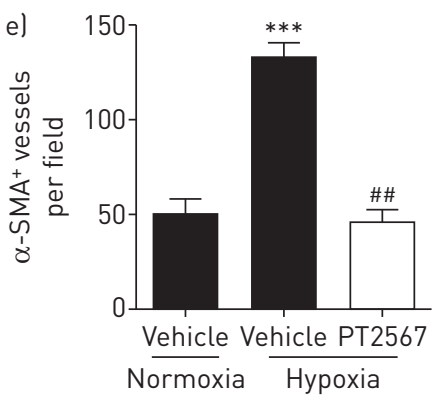

FIGURE 4 Small molecule hypoxia inducible factor 2 inhibitor PT2567 significantly attenuates development of hypoxia-induced pulmonary hypertension at 4 weeks in adult rats. mPAP: mean pulmonary arterial pressure; CCNA1: cyclin A1; TNC: tenascin C. Sprague Dawley male rats weighing 210-245 g (Charles River Laboratories, Wilmington, MA, USA) were housed in chambers under normoxia or hypoxic (simulated altitude of $\sim 18000 \mathrm{ft}(\sim 5500 \mathrm{~m}))$ conditions for 4 weeks. Rats were dosed with vehicle methylcellulose $(0.5 \%) /$ Tween-80 (0.5\%) or PT2567 (300 mg. $\mathrm{kg}^{-1} \cdot \mathrm{day}^{-1}$ ) beginning the day they were placed in chambers. After 4 weeks, end-point measurements for the experimental animals were conducted. a) mPAP. b) Ratio of the weight of the right ventricle to body weight $\left(\right.$ weight $_{\mathrm{RV}} /$ weight $_{\text {Body }}$ ). c) Representative images of pulmonary vessels stained with anti-macrophage/monocyte antibody clone ED-1 (top), anti-Ki-67 antibody (middle) or anti-TNC antibody (bottom). The levels of Cd68, Ccna1 and Tnc mRNAs in the whole lung tissues of indicated rats are also shown (right). d, e) $\alpha$-SMA-positive pulmonary vessels. Scale bar: $100 \mu \mathrm{m}$. *: $p<0.05$; ***: $p<0.001$, difference between hypoxia versus normoxia in the same genotype (or treatment) group; ${ }^{\# \#: ~} p<0.01$; \#\#\#: $p<0.001$, difference between genotypes or treatments under hypoxic conditions; ११: $p<0.01$, difference between genotypes or treatments under normoxic conditions. Statistical significance determined by two-way ANOVA $(a, b)$ or the t-test (c-e). 

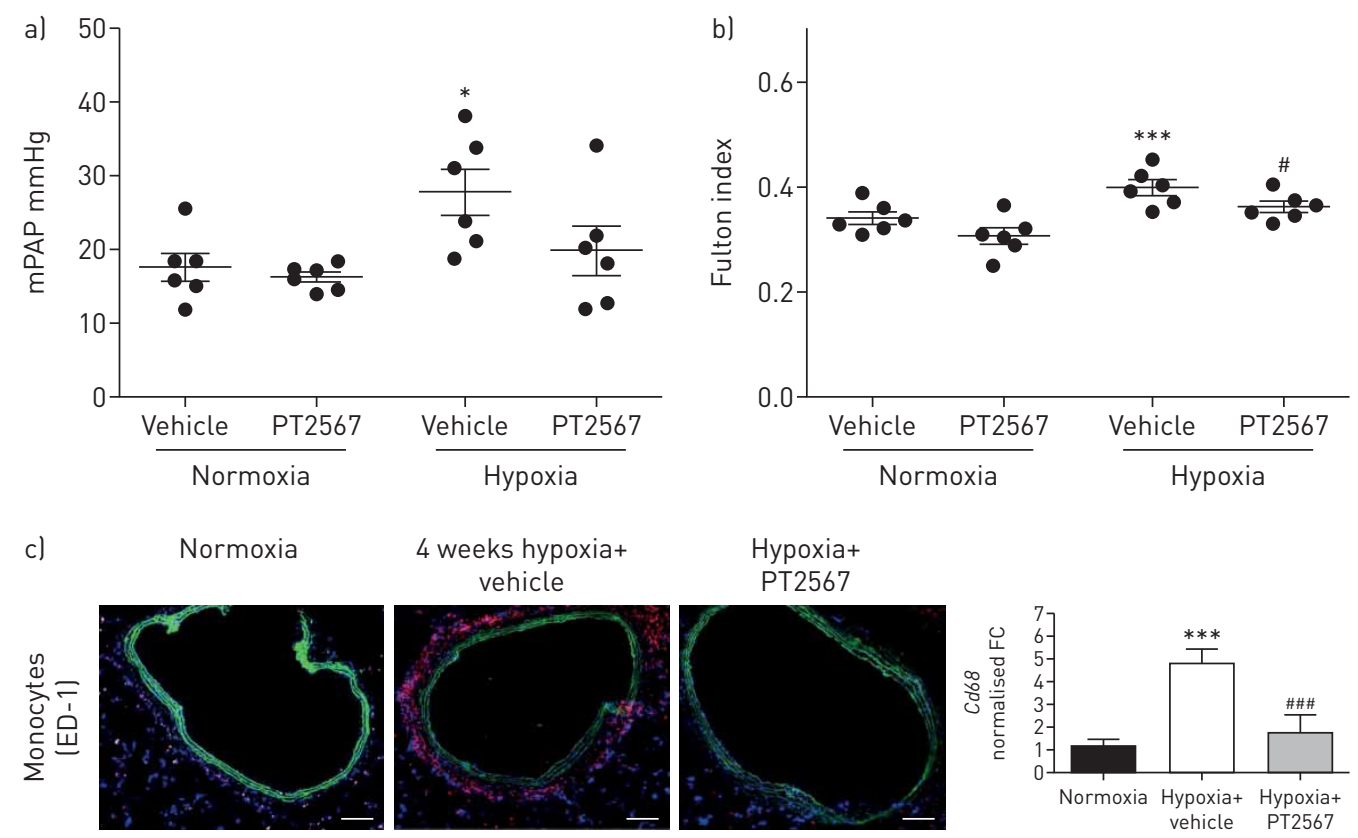

4 weeks hypoxia+ vehicle
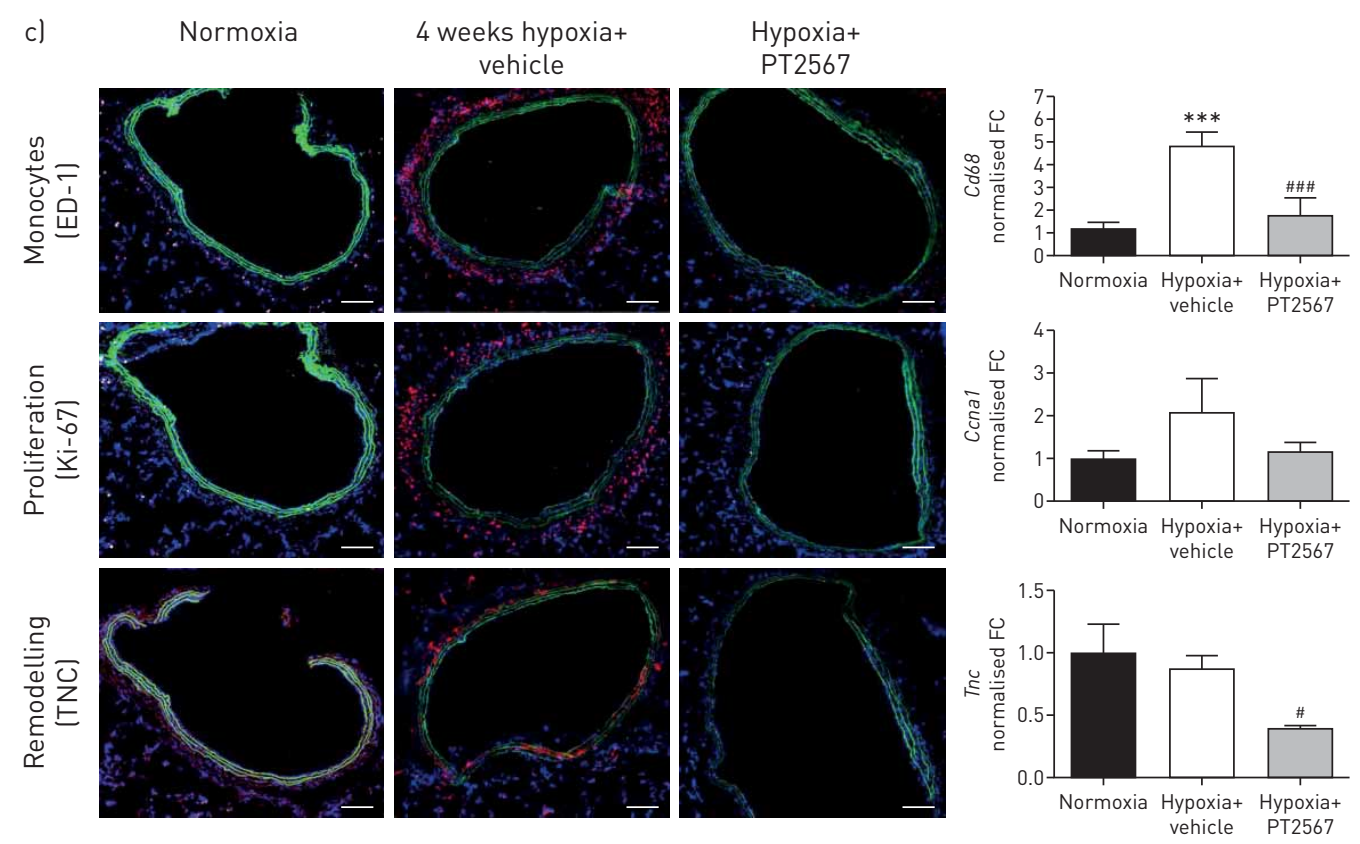

FIGURE 5 Small molecule hypoxia inducible factor 2 inhibitor PT2567 significantly attenuates early events in hypoxia-exposed adult rats at 4 days. mPAP: mean pulmonary arterial pressure; CCNA1: cyclin A1; TNC: tenascin C. Sprague Dawley rats were housed in chambers under normoxia or hypoxic conditions for 4 days and dosed with vehicle or PT2567 as described in figure 4. After 4 days, end-point measurements for the experimental animals were conducted. al mPAP. b) Ratio of the weight of the right ventricle to body weight (weight $\mathrm{RV}_{\mathrm{RV}}$ /weight $\mathrm{Body}_{\mathrm{f}}$. c) Representative images of pulmonary vessels stained with anti-macrophage/monocyte antibody clone ED-1 (top), anti-Ki-67 antibody (middle) or anti-TNC antibody (bottom). The levels of Cd68, Ccna1 and Tnc mRNAs in lungs of indicated rats are also shown (right). Scale bar: $100 \mu \mathrm{m} .{ }^{*}: p<0.05$; ${ }^{* * *}$ : $p<0.001$, difference between hypoxia versus normoxia in the same genotype (or treatment) group; ${ }^{\#}: p<0.05$; \#\#\#: $p<0.001$, difference between genotypes or treatments under hypoxic conditions. Statistical significance determined by two-way ANOVA $(a, b)$ or the t-test (c).

exposed to 4 days of hypoxia (supplementary figure S4). Importantly, the HIF2 inhibitor PT2567 reduced expression of these genes (supplementary figure S4). However, Pdgfb and Cxcr4 were induced, and Id 1 was reduced, only in rats exposed to short-term hypoxia (supplementary figure S4). The effectiveness of the HIF2 inhibitor in preventing monocyte/macrophage accumulation and vascular cell proliferation, and the fact that changes in gene expression were largely overlapping in rats exposed to short-term hypoxia and to long-term hypoxia, support the idea that HIF2 activity is essential in initiating hypoxia pulmonary hypertension at a very early stage (4 days).

\section{Normal human pulmonary artery ECs display unique responses to acute hypoxia in a HIF2 $\alpha$-dependent manner}

Our short-term in vivo hypoxia studies support the hypothesis that HIF2 activity is activated by hypoxia very early in one or multiple pulmonary vascular cells (ECs, SMCs and fibroblasts), initiating pulmonary hypertension development. Here, we attempted to determine the main cell type(s) whose activation by acute hypoxia may explain the observed events in rats exposed to 4 days of hypoxia (figure 5) by first 
comparing and contrasting the response of normal human pulmonary artery-derived ECs, SMCs and fibroblasts to acute hypoxia. We chose to use normal vascular cells as we were studying the function of HIF in pulmonary hypertension initiation. Acute hypoxia $(1.5 \%$ for $16 \mathrm{~h})$ significantly activated expression of several classic HIF target genes including ADM, CA9, GLUT1, NDRG1 and VEGFA in all three cell types, suggesting activation of these classical HIF target genes lacks cell type specificity (figure 6a). Hypoxic induction of the pro-inflammatory genes CXCR4, SDF1 and ICAM1 was most prominent in ECs (figure 6b). Additional unique responses of ECs to acute hypoxia was evidenced by reduced expression of ID1 and ID3, and increased expression of the growth factor TGFA, a ligand of EGFR (figure 6c), all of which can promote cell proliferation and survival. However, induction of APLN, EDN1, PDGFB and TSP1 was mainly observed in SMCs and fibroblasts, while ARG1 and ARG2 were not induced by acute hypoxia in any of the three cell types (figure $6 \mathrm{c}$ ). These data support a unique role of ECs in response to acute hypoxia, to increase genes involved in inflammation and cell migration and proliferation. Thus, we determined if HIF2 is responsible for increased expression of inflammatory and proliferative genes in ECs under hypoxia. Interestingly, HIF2 inhibitor PT2567 completely abolished hypoxia-mediated changes of inflammatory (CXCR4, ICAM1 and SDF1) (figure 7b) and signalling/proliferation (ID1, ID3 and TGFA) (figure 7c) genes in control ECs, but only attenuated hypoxic induction of classical HIF target genes (figure 7a). The essential role of HIF2 (but not HIF1) in regulating inflammatory and signalling/ proliferation genes in control ECs was further confirmed using a small interfering RNA (siRNA) approach as HIF2A siRNA (but not HIF1A siRNA) significantly attenuated changes of inflammatory and signalling/ proliferation gene expression, induced by hypoxia in ECs (supplementary figure S5c and $\mathrm{d}$ ). While the direct function of proteins such as ID1 and ID3 is likely intracellular, the increased production of genes/ proteins such as stromal cell-derived factor $1(S D F 1)$ and tumour necrosis factor- $\alpha$ (TGFA) in ECs could also involve paracrine signalling. To assess the role of activated ECs in regulating other pulmonary vascular cells, we prepared conditional medium from normal ECs cultured under normoxia or hypoxia, in the presence or absence of a HIF2 inhibitor PT2567. We found that HIF2-mediated activation of control ECs also increased the expression of genes involved in cell proliferation (CCNE1 and CCNE2), pro-inflammation (CCL2) and anti-apoptosis (BCL2, BCL2L1 and BIRC5) in control SMCs. This suggests a role for HIF2 signalling in activating ECs, which promote SMC activation (supplementary figure S6). Thus, our studies support a hypothesis that activation of ECs by hypoxia in a HIF2-dependent manner (but not HIF1) activates ECs, also initiating proliferation of other vascular cells (SMCs) and recruitment of monocytes/macrophages via increased expression of inflammatory cytokines (SDF1) and growth factors (TGFA).

\section{Hif2a expression in ECs is required for hypoxia-induced pulmonary hypertension and vascular remodelling in mice}

To confirm the essential role of EC Hif2a in pulmonary hypertension development in vivo and to better understand the role of EC HIF2 in pulmonary hypertension development, we generated and exposed EC Hif $2 a$ knockouts and their controls to normoxia or hypoxia for 5 weeks. As expected, Hif $2 a^{f l f f l}$-EC-Cre ${ }^{-}$ animals subjected to hypoxia displayed an increase in Hct (figure 8a) and pulmonary hypertension development (figure 8b-d). We observed a similar increase of Hct in the hypoxia-exposed Hif $2 a^{f l f l}$-EC-Cre ${ }^{+}$mice, confirming previous reports that endothelial HIF2 $\alpha$ is not required for hypoxia-induced erythropoiesis and erythrocytosis [4]. However, hypoxia-exposed Hif $2 a^{f l f l}$-EC-Cre ${ }^{+}$mice exhibited no signs of pulmonary hypertension (figure $8 \mathrm{~b}-\mathrm{d}$ ). The increase in the number of $\alpha$-smooth muscle actin-positive pulmonary vessels in hypoxia-exposed $H i f 2 a^{f l f f l}$-EC-Cre ${ }^{-}$mice was also abolished in hypoxia-exposed Hif2 $a^{f l f l}$-EC-Cre ${ }^{+}$mice (figure $8 \mathrm{e}$ and $\mathrm{f}$ ). We also examined the same set of genes that we studied in rats treated with the HIF2 inhibitor (supplementary figure S3). As expected, genes induced by hypoxia in the lungs of control rats (supplementary figure S3) were partially overlapping with the genes induced in mice by chronic hypoxia (supplementary figure S7). The list included Ndrg1, Sdf1, Arg1 and $E d n 1$. However, the only genes that were reduced in both hypoxia-exposed rats treated with HIF2 inhibitor (supplementary figure S3) and in mice with EC Hif2a knockout were Sdf1, Arg1 and Edn1 (supplementary figure S7). This indicates the particular importance of these genes in the development of hypoxia-induced pulmonary hypertension. Interestingly, we found that Actal and $M y h 7$ gene expression was markedly reduced in the right ventricle of hypoxic Hif $2 a^{f l / f l}-\mathrm{EC}-\mathrm{Cre}^{+}$mice (supplementary figure S8) compared with the right ventricle of hypoxic Hif $2 a^{f l f l}$-EC-Cre ${ }^{-}$mice, confirming reduced right ventricle remodelling.

\section{Discussion}

Using both genetic and pharmacological approaches to inhibit global HIF1 or HIF2 activity, we found that inhibition of HIF2 $\alpha$ (but not HIF1 $\alpha$ ) attenuates the development of pulmonary hypertension in normal adult animals exposed to chronic (4-5 weeks) hypoxia. Importantly, in addition to addressing the role of HIF1 and HIF2 in hypoxia-induced pulmonary hypertension development using normal adult animals, we also uncovered several novel roles of HIF2 in adult animals, including its requirement for animals to 

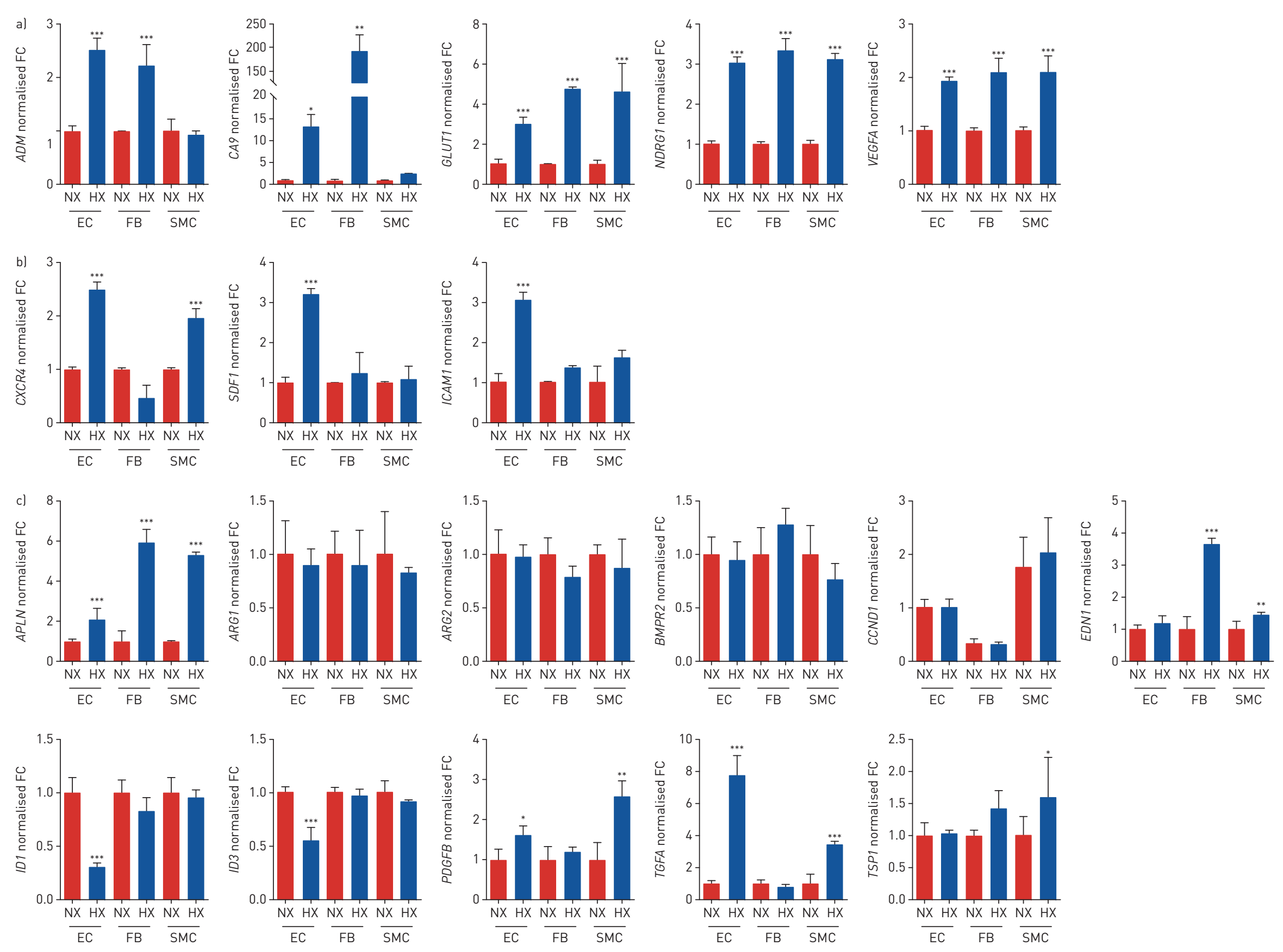
FIGURE 6 Normal human pulmonary artery vascular cells exhibit unique properties in response to acute hypoxia. FC: fold change; EC: endothelial cell; FB: fibroblast; SMC: smooth muscle cell. Normal human pulmonary artery vascular cells (ECs, FBs and SMCs; $\mathrm{n}=5$ for each cell type) were cultured under normoxia (NX) or hypoxia $\left(\mathrm{HX} ; 1.5 \% \mathrm{O}_{2}\right)$ for $16 \mathrm{~h}$ and then cells were collected for RNA preparation. The same set of genes examined in vivo was studied here. Results were from at least five different cell populations for each cell type, in which the result for a specific cell population was from three independent normoxia or hypoxia experiments here or other similar experiments in this paper. al Classical hypoxia inducible factor target genes. b) Genes involved in inflammation. c) Genes involved in signalling. ${ }^{*}: p<0.05 ;{ }^{* *}: p<0.01 ;{ }^{* * *}: p<0.001$, difference between hypoxia versus normoxia in the same genotype (or treatment) group. Statistical significance determined by the t-test.

survive under chronic hypoxic conditions. Our in vitro studies (pulmonary vascular cell response to acute hypoxia), in combination with short-term hypoxia in vivo studies, support a hypothesis that EC HIF2 is "essential" in pulmonary hypertension initiation because only ECs can increase the production of diffusible cytokines (SDF1), which may recruit monocytes/macrophages and other blood/bone marrow-derived cells to lung vessels, at early stages of pulmonary hypertension development. This result is
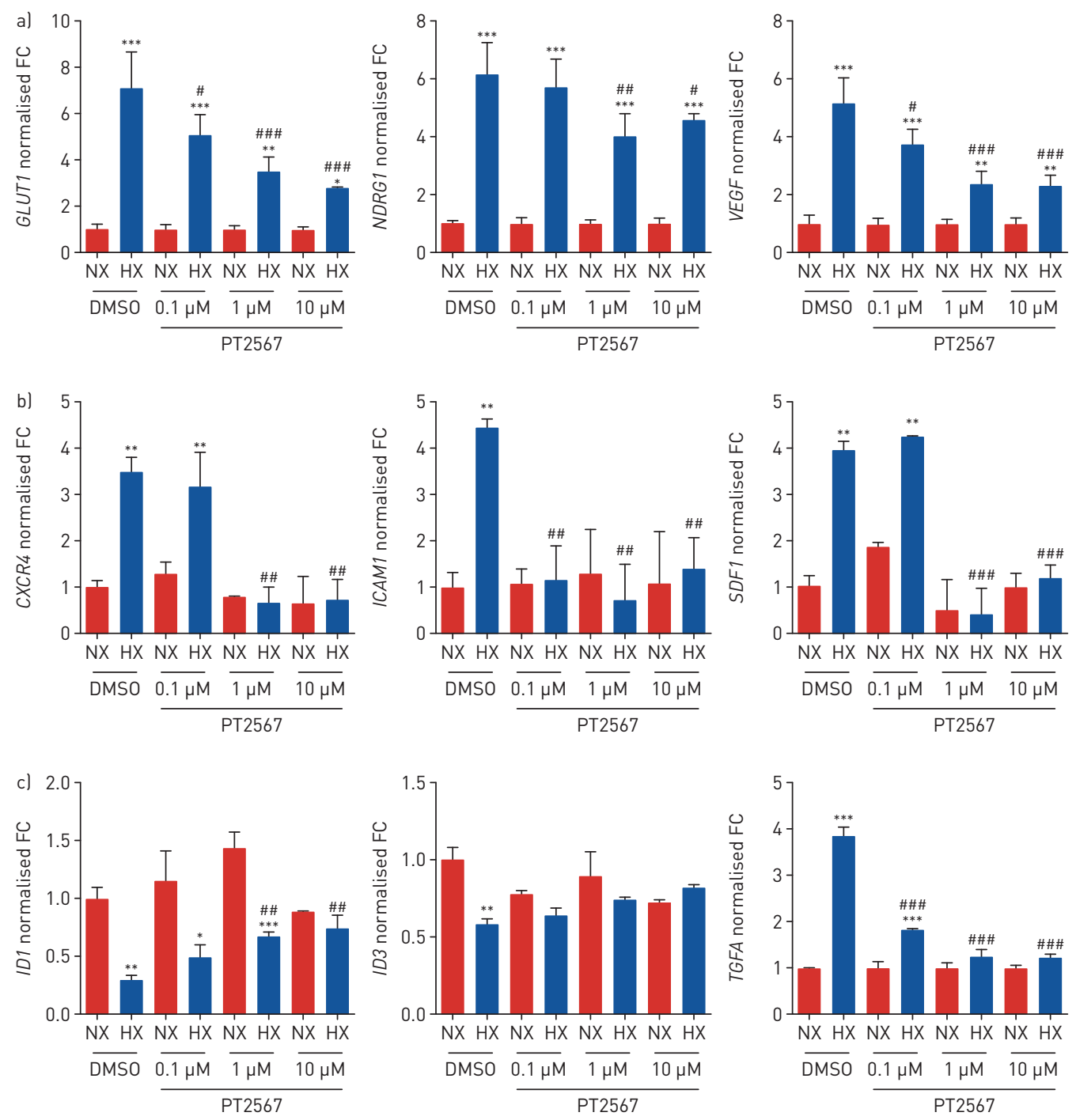

FIGURE 7 Hypoxia inducible factor-2 (HIF2) inhibitor PT2567 significantly attenuates altered production of genes involved in inflammation and signalling in normal pulmonary endothelial cells (ECs) in response to acute hypoxia. FC: fold change; DMSO: dimethyl sulfoxide. To determine if HIF2 activity is responsible for hypoxia-mediated gene expression changes in ECs, normal human pulmonary artery ECs $(n=3)$ were cultured under normoxia (NX) or hypoxia $\left(\mathrm{HX} ; 1.5 \% \mathrm{O}_{2}\right)$ for $16 \mathrm{~h}$ in the presence of DMSO (control) or different concentrations of HIF2 inhibitor PT2567, and then cells were collected for RNA preparation and quantitative real-time PCR. al Select classical HIF target genes. b) Genes involved in inflammation that are significantly induced by hypoxia in ECs (figure 6b). c) Genes involved in signalling that are significantly altered by hypoxia in ECs (figure 6c). ${ }^{*}: p<0.05 ;{ }^{* *}$ : $p<0.01 ;{ }^{* * *}$ : $p<0.001$, difference between hypoxia versus normoxia in the same genotype (or treatment) group; ${ }^{\#}: p<0.05 ;{ }^{\# \#}: p<0.01$; ${ }^{\# \# \#}: p<0.001$, difference between genotypes or treatments under hypoxic conditions. Statistical significance determined by the t-test. 

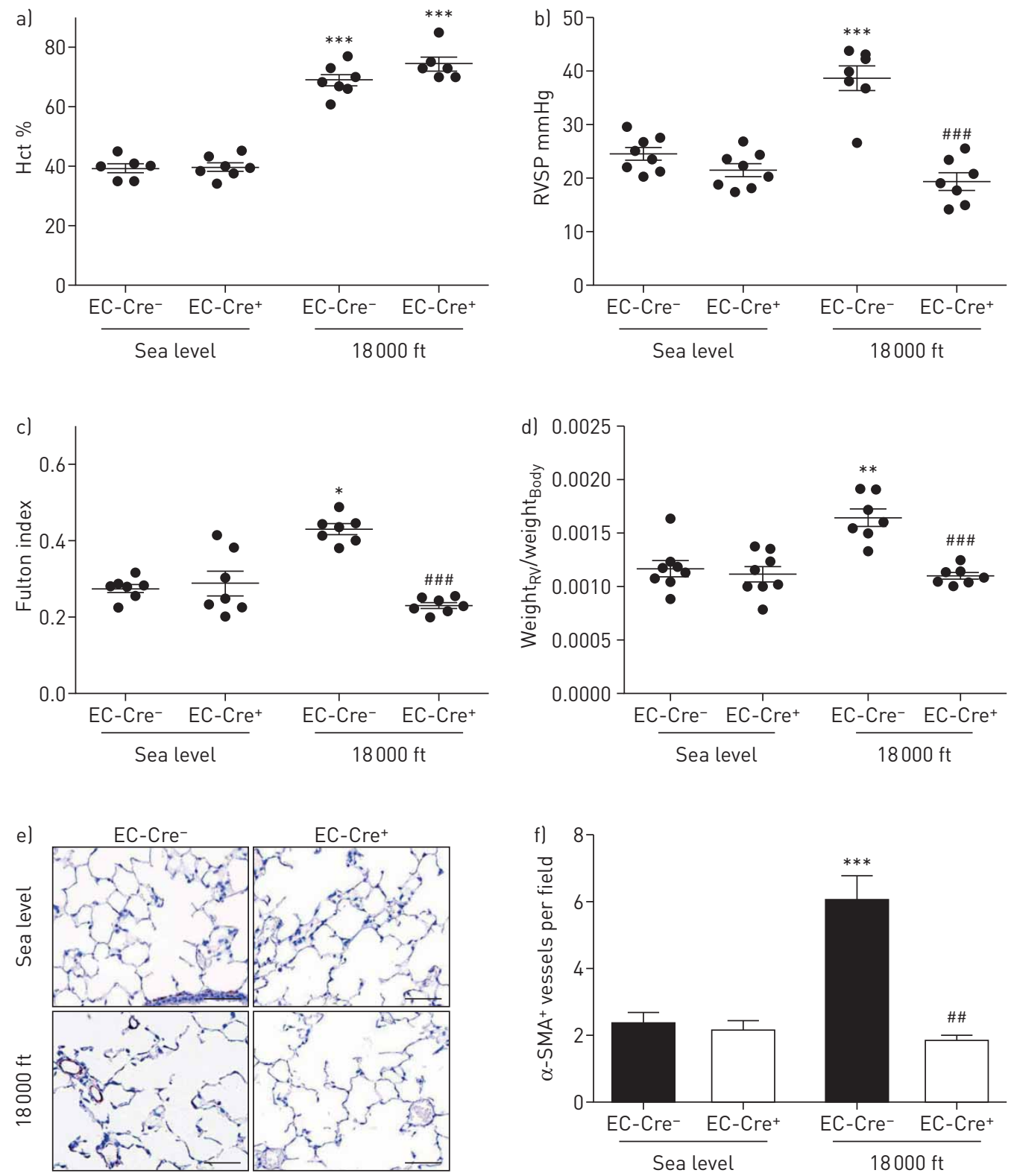

FIGURE 8 Hif2a expression in endothelial cells (ECs) is required for the development of hypoxia-induced pulmonary hypertension. Hct: haematocrit; RVSP: right ventricular systolic pressure; $\alpha$-SMA: $\alpha$-smooth muscle actin. Hif2a knockout or Hif2a wild-type mice were exposed to either normoxia (sea level) or hypoxia (simulated altitude of $\sim 18000 \mathrm{ft}(\sim 5500 \mathrm{~m})$ ) for 5 weeks. a-f) End-point measurements in these mice: a) Hct,

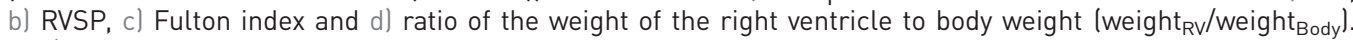
e, f) $\alpha$-SMA-positive pulmonary vessels. Scale bar: $100 \mu \mathrm{m} .{ }^{*}$ : $p<0.05$; ${ }^{* *}$ : $p<0.01$; ${ }^{* * *}$ : $p<0.001$, difference between hypoxia versus normoxia in the same genotype lor treatment) group; ${ }^{\# \#}: p<0.01 ; \# \#$ : $p<0.001$, difference between genotypes or treatments under hypoxic conditions. Statistical significance determined by two-way ANOVA (a-d) or the t-test ( $f$ ).

also consistent with an earlier study showing that EC-derived SDF1 contributes to pulmonary hypertension in PHD2-deficient mice [3]. Our studies also support the hypothesis that HIF2 in SMCs or fibroblasts is "not essential" in pulmonary hypertension initiation. This might be due to the fact that more than one cell type (among SMCs, fibroblasts and ECs) can induce the expression of the pulmonary hypertension-related genes such as APLN, EDN1, PDGFB, TGFA and TSP1. Thus, our studies contribute to a better understanding of the role of HIF2 in pulmonary hypertension initiation.

Pulmonary hypertension is observed in post-natal humans and animals with normal pulmonary circulation in response to chronic hypoxia stress. However, to study the role of HIF in pulmonary hypertension development, most published studies used models in which HIF deletion or HIF activation (in PHD2 
knockout) was initiated in embryonic life $[1,3-8,10]$. We think results from such approaches need to be re-evaluated because 1) all these models are known to exhibit vascular defects in developing embryos and likely in adult mice derived from these embryos [12-17], and 2) there are examples demonstrating differences in phenotype (baseline as well as stressed) when gene deletion is initiated in the embryo versus in the adult [27-29]. For example, inhibition of monocyte chemoattractant protein 1 (MCP1) initiated in adult animals reduces hypoxia- or monocrotaline-induced pulmonary hypertension $[27,28]$. In contrast, MCP1 or MCP1 receptor knockout mice (initiated in the embryo) exhibit spontaneous pulmonary hypertension [29]. Our data showing the effects of HIF2 inhibition in normal adult animals demonstrate the functional importance of HIF2 in pulmonary hypertension development, eliminating the concerns that HIF2's function in most previous reported studies is due to the defects in the lung vasculature and heart by Hif $2 a$ deletion or activation in developing embryos. Although we also used mice with constitutive EC-specific knockout of Hif $2 a$, initiated in the embryo, we recognise that results from the EC Hif $2 a$ knockout study have limitations. However, the purpose of our EC Hif2a knockout study is not to determine the general function of HIF2 in hypoxia-induced pulmonary hypertension, but to provide a molecular explanation for the function of HIF2 in ECs in pulmonary hypertension development. Results from in vivo studies in these mice confirmed 1) results obtained from mice with an inducible, global knockout, 2) results from animals treated with two different inhibitors and 3) results from in vitro studies.

The role of HIF1 $\alpha$ in the development of hypoxia-induced pulmonary hypertension is controversial: studies demonstrated either a partial amelioration of pulmonary hypertension [10,11], or a temporary slowing of pulmonary hypertension progression [1] or even elevated PAPs [2], in mice with reduced Hifla expression. Additional in vivo studies in which HIF1 is activated by genetic manipulation of PHDs or the von Hippel-Lindau (VHL) protein demonstrated HIF1 $\alpha$ was not required for pulmonary hypertension development $[3,4,9]$. We speculate that the different results reported could be due to using hypoxia or pseudo-hypoxia (PHD or VHL knockout animals) approaches or deletion of Hifla in different cell types or deletion of HIF1 $\alpha$ initiated in embryos or adult mice. Our studies using global (not cell-type-specific) inhibition of HIF1 $\alpha$ in normal adult animals (not initiated in embryo) and using hypoxia to activate HIF (not pseudo-hypoxia) do not support an indispensable role of HIF1 $\alpha$ for pulmonary hypertension in animals after 4-5 weeks of hypoxia exposure. However, we cannot exclude a transient role of HIF1 $\alpha$ in the earlier stage of pulmonary hypertension development, which we did not investigate. Indeed, a transient role of HIF1 $\alpha$ in hypoxia-induced pulmonary hypertension has been reported [1]. Also, we cannot exclude disease-relevant HIF1 $\alpha$ signalling in SMCs in vivo, as we did not confirm sufficient knockdown in this cell type in our ASO and inducible knockout systems. Lastly, our in vivo studies were performed using hypobaric hypoxia, while previous studies were performed using normobaric hypoxia. Since some physiological responses differ depending on the mode of hypoxia [30], the different experimental setting might also contribute to some of the differences between our findings and previous studies on the role of HIF1 $\alpha$ in hypoxic pulmonary hypertension.

Another distinct feature of this study is to inhibit global, not cell-type-specific HIF activity for the purpose of determining HIF function in pulmonary hypertension development. Cell-type-specific knockout is a powerful method to understand the contribution of the targeted gene in a specific cell type to the disease. However, we believe conclusions derived solely from cell-type-specific knockout experiments may have shortcomings for the purpose of determining the general function of a gene in a disease such as pulmonary hypertension where interactions between multiple cell types are clearly necessary for disease progression and a specific gene may have different or opposite functions in different cell types involved in the disease. For example, caveolin-1 is reduced in pulmonary hypertension ECs and its reduction in ECs promotes pulmonary hypertension development [31]; however, caveolin-1 is overexpressed in pulmonary hypertension SMCs and its overexpression in SMCs also promotes pulmonary hypertension development $[31,32]$. Using global HIF gene deletion or pharmacological inhibition, we concluded that HIF2 (but not HIF1) is important for hypoxia-induced pulmonary hypertension development. However, EC Hif $2 a$ deletion (figure 8) appears to be more effective than global HIF2 reduction (ASO and HIF2 inhibitor PT2567) in preventing development of hypoxia-induced pulmonary hypertension, suggesting the true role of HIF2 in hypoxia-induced pulmonary hypertension development is less important than reported in EC Hif $2 a$ deletion models.

Cell-type-specific knockout approaches may also miss the possible side-effects of therapeutic inhibition of a gene such as Hif2a, especially if Hif $2 a$ also plays important roles in other cell types for other processes. Indeed, complete global deletion of Hif2a is detrimental for survival of mice under chronic hypoxia, which is a novel and important finding. Although the underlying causes for this lethality and the different tolerance to chronic hypoxia between Hif $2 a$ knockout mice and mice/rats treated with Hif $2 a$ inhibitor are beyond the scope of this study, we speculate that mice with significant loss of Hif $2 a$ might have succumbed to cardiogenic shock under hypoxic conditions. This is based on our findings that 
Hif2a-ASO treatment reduced levels of circulating catecholamines that were significantly increased in hypoxia in control-ASO mice (supplementary figure S2b). Hif2a-ASO also abolished the increase in heart rate that was observed in control mice with hypoxia exposure (supplementary figure S2c), resulting in reduced cardiac output (supplementary figure S2d). Hif2a knockdown also resulted in lower $\mathrm{d} P / \mathrm{d} t_{\max }$ (right ventricle), a parameter of ventricular systolic function (supplementary figure S2e). These effects could be mediated by loss of Hif2a in heart tissue (supplementary figure S2a) or by loss in other organs (e.g. the adrenal glands). In conclusion, we speculate that lower Hct in Hif2a-ASO-treated animals, in addition to the observed changes in cardiac function, could result in critically low delivery of oxygen and finally death of Hif2a knockout mice. This interpretation of our data is consistent with previous studies demonstrating Hif2a-dependent changes in catecholamines [8], heart rate, and cardiac output and physiology [33-35]. Importantly, we did not observe changes in heart rate in mice with EC-specific Hif2a knockout, demonstrating that the observed reduction in RVSP in Hif2a-ASO-treated animals was not merely due to impaired cardiac function. In summary, our study suggests that we need to exercise caution, particularly for patients residing at high altitude, if a HIF $2 \alpha$ inhibitor is going to be used in the clinic in the future.

Most published studies have evaluated the end-effect of HIF inhibition in animals exposed to chronic hypoxia without knowledge of the role of HIF in the early stage of pulmonary hypertension initiation. We performed studies in animals exposed to chronic as well as short-term hypoxia. We revealed that HIF2 is essential in several early events (macrophage recruitment and vascular cell proliferation) of pulmonary hypertension development, likely by activating genes such as those for Sdf1 (inflammation) and $\mathrm{Tg} f a$ (cell proliferation) in vivo.

To the best of our knowledge, this is the first time that the responses of the three primary normal human pulmonary vascular cell types (ECs, SMCs and fibroblasts) to acute hypoxia have been examined concurrently for a set of genes that have been reported $[3,4,6,9,22-24]$ and demonstrated in our current studies (supplementary figures S3, S4 and S7) to be important for pulmonary hypertension development. Our studies lead to novel findings that the EC is the primary cell type that can be activated by short-term hypoxia (ID reduction), in a HIF2-dependent manner to produce inflammatory (SDF1) and growth promoting factors (TGFA) (figure 7), to activate other vascular cells (supplementary figure S6). Our findings from short-term in vivo and in vitro studies are consistent with previous studies by us and others that recruitment of bone marrow/blood-derived cells such as monocytes/macrophages is an early and critical event in pulmonary hypertension development [25, 36-39], and cytokines/chemokines including SDF1 are among the earliest inflammatory factors increased in the pulmonary arteries of hypoxia-exposed animals and whose increase precedes monocyte/macrophage accumulation [26]. Our in vitro studies also indicated that normal SMCs and fibroblasts can be acutely activated by hypoxia to produce factors such as APLN, EDN1 and TSP1 that have been shown to be involved in vessel constriction $[4,6]$. While knockout of HIF2A in SMCs does not prevent hypoxic pulmonary hypertension [7], studies on the effect of HIF2A inhibition in fibroblasts, in combination with other pulmonary cell types, such as SMCs, are needed. Furthermore, our data indicated that there is no hypoxic induction of ARG1 and ARG2 in normal ECs, SMCs and fibroblasts (figure 6), although we consistently observed increased expression of Arg1 and Arg2 in lungs of chronic and short-term hypoxia-exposed rodents and whose expression is reduced by HIF2 inhibition (supplementary figures S3, S4 and S7). These data suggest that increased expression of genes such as those for ARG1 and ARG2 could be mainly from recruited cells.

The critical role of HIF2 in the development of hypoxia-induced pulmonary hypertension has raised significant interest in targeting HIF2 for the treatment of pulmonary hypertension patients. In fact, one study has already demonstrated a quite effective role of a HIF2 inhibitor in reversing pulmonary hypertension in animal models [40]. However, small animal models for pulmonary hypertension have well-recognised limitations [41]. More importantly, targets that have been demonstrated to be effective in animal models often fail in clinical trials $[42,43]$. Thus, we believe further studies are needed before initiation of a HIF2 inhibitor clinical trial in humans. Accumulating data indicate that vascular cells established from pulmonary hypertension patients and large animals (cows) exhibit and maintain their unique phenotypes in vitro [44]. We believe these cells could provide an excellent platform to further determine the role of HIF2 for pulmonary hypertension treatment. In addition, although HIF1 is not essential in hypoxia-induced pulmonary hypertension development, the role of HIF1 in pulmonary hypertension disease maintenance is also possible.

In summary, our studies have demonstrated a positive role of HIF2 in pulmonary hypertension development in response to chronic hypoxia in normal adult animals. However, more research is needed to determine if HIF2 is truly a good pulmonary hypertension treatment target because HIF2 activity is likely to be one of the many factors that are required, acting in different cell types, at different stages, to initiate pulmonary hypertension [45]. In addition, there are data from cancer research that factors/ 
pathways that initiate cancer often fail to be good treatment targets because there are many additional changes that occur during cancer progression.

Acknowledgements: Ionis Pharmaceuticals (Carlsbad, CA, USA) supplied Hif1-ASOs and technical support.

Conflict of interest: C-J. Hu has nothing to disclose. J.M. Poth has nothing to disclose. H. Zhang has nothing to disclose. A. Flockton has nothing to disclose. A. Laux has nothing to disclose. S. Kumar has nothing to disclose. B. McKeon has nothing to disclose. G. Mouradian has nothing to disclose. M. Li has nothing to disclose. S. Riddle has nothing to disclose. S.C. Pugliese has nothing to disclose. R.D. Brown has nothing to disclose. E.M. Wallace has a patent "Compositions for Use in Treating Pulmonary Arterial Hypertension" pending to Peloton Therapeutics. B.B. Graham reports grants from National Institutes of Health, during the conduct of the study. M.G. Frid has nothing to disclose. K.R. Stenmark reports personal fees for advisory board or steering committee work from Pfizer, New York, Actelion (Entelligence) and Janssen Research and Development, outside the submitted work.

Support statement: This work was supported by the US Dept of Defense (grant PR140977) and the National Heart, Lung, and Blood Institute (grant P0 HL014985). Funding information for this article has been deposited with the Crossref Funder Registry.

\section{References}

1 Yu AY, Shimoda LA, Iyer NV, et al. Impaired physiological responses to chronic hypoxia in mice partially deficient for hypoxia-inducible factor $1 \alpha$. J Clin Invest 1999; 103: 691-696.

2 Kim YM, Barnes EA, Alvira CM, et al. Hypoxia-inducible factor- $1 \alpha$ in pulmonary artery smooth muscle cells lowers vascular tone by decreasing myosin light chain phosphorylation. Circ Res 2013; 112: 1230-1233.

3 Dai Z, Li M, Wharton J, et al. Prolyl-4 hydroxylase 2 (PHD2) deficiency in endothelial cells and hematopoietic cells induces obliterative vascular remodeling and severe pulmonary arterial hypertension in mice and humans through hypoxia-inducible factor-2 $\alpha$. Circulation 2016; 133: 2447-2458.

4 Kapitsinou PP, Rajendran G, Astleford L, et al. The endothelial prolyl-4-hydroxylase domain 2/hypoxia-inducible factor 2 axis regulates pulmonary artery pressure in mice. Mol Cell Biol 2016; 36: 1584-1594.

5 Labrousse-Arias D, Castillo-Gonzalez R, Rogers NM, et al. HIF-2 $\alpha$-mediated induction of pulmonary thrombospondin-1 contributes to hypoxia-driven vascular remodelling and vasoconstriction. Cardiovasc Res 2016; 109: 115-130.

6 Cowburn AS, Crosby A, Macias D, et al. HIF2 $\alpha$-arginase axis is essential for the development of pulmonary hypertension. Proc Natl Acad Sci USA 2016; 113: 8801-8806.

7 Tang H, Babicheva A, McDermott KM, et al. Endothelial HIF- $2 \alpha$ contributes to severe pulmonary hypertension due to endothelial-to-mesenchymal transition. Am J Physiol Lung Cell Mol Physiol 2018; 314: L256-LL75.

8 Brusselmans K, Compernolle V, Tjwa M, et al. Heterozygous deficiency of hypoxia-inducible factor- $2 \alpha$ protects mice against pulmonary hypertension and right ventricular dysfunction during prolonged hypoxia. J Clin Invest 2003; 111: 1519-1527.

9 Hickey MM, Richardson T, Wang T, et al. The von Hippel-Lindau Chuvash mutation promotes pulmonary hypertension and fibrosis in mice. J Clin Invest 2010; 120: 827-839.

10 Ball MK, Waypa GB, Mungai PT, et al. Regulation of hypoxia-induced pulmonary hypertension by vascular smooth muscle hypoxia-inducible factor-1 $\alpha$. Am J Respir Crit Care Med 2014; 189: 314-324.

11 Sheikh AQ, Saddouk FZ, Ntokou A, et al. Cell autonomous and non-cell autonomous regulation of SMC progenitors in pulmonary hypertension. Cell Rep 2018; 23: 1152-1165.

12 Ema M, Taya S, Yokotani N, et al. A novel bHLH-PAS factor with close sequence similarity to hypoxia-inducible factor $1 \alpha$ regulates the VEGF expression and is potentially involved in lung and vascular development. Proc Natl Acad Sci USA 1997; 94: 4273-4278.

13 Kotch LE, Iyer NV, Laughner E, et al. Defective vascularization of HIF-1 $\alpha$-null embryos is not associated with VEGF deficiency but with mesenchymal cell death. Dev Biol 1999; 209: 254-267.

14 Favier J, Kempf H, Corvol P, et al. Coexpression of endothelial PAS protein 1 with essential angiogenic factors suggests its involvement in human vascular development. Dev Dyn 2001; 222: 377-388.

15 Compernolle V, Brusselmans K, Acker T, et al. Loss of HIF- $2 \alpha$ and inhibition of VEGF impair fetal lung maturation, whereas treatment with VEGF prevents fatal respiratory distress in premature mice. Nat Med 2002; 8: $702-710$.

16 Takeda K, Ho VC, Takeda H, et al. Placental but not heart defects are associated with elevated hypoxia-inducible factor $\alpha$ levels in mice lacking prolyl hydroxylase domain protein 2. Mol Cell Biol 2006; 26: 8336-8346.

17 Gnarra JR, Ward JM, Porter FD, et al. Defective placental vasculogenesis causes embryonic lethality in VHL-deficient mice. Proc Natl Acad Sci USA 1997; 94: 9102-9107.

18 Wallace EM, Rizzi JP, Han G, et al. A small-molecule antagonist of HIF2 $\alpha$ is efficacious in preclinical models of renal cell carcinoma. Cancer Res 2016; 76: 5491-5500.

19 Wehn PM, Rizzi JP, Dixon DD, et al. Design and activity of specific hypoxia-inducible factor-2 $\alpha$ (HIF-2 $\alpha)$ inhibitors for the treatment of clear cell renal cell carcinoma: discovery of clinical candidate (S)-3-((2,2-difluoro-1-hydroxy-7-(methylsulfonyl)-2,3-dihydro-1H-inden-4-yl)oxy)-5-fluorobenzonitrile (PT2385). J Med Chem 2018; 61: 9691-9721.

20 Courtney KD, Infante JR, Lam ET, et al. Phase I dose-escalation trial of PT2385, a first-in-class hypoxia-inducible factor- $2 \alpha$ antagonist in patients with previously treated advanced clear cell renal cell carcinoma. J Clin Oncol 2018; 36: $867-874$.

21 Shin MK, Drager LF, Yao Q, et al. Metabolic consequences of high-fat diet are attenuated by suppression of HIF-1 $\alpha$. PLoS One 2012; 7: e46562.

22 Stenmark KR, Fagan KA, Frid MG. Hypoxia-induced pulmonary vascular remodeling: cellular and molecular mechanisms. Circ Res 2006; 99: 675-691.

23 Pugliese SC, Poth JM, Fini MA, et al. The role of inflammation in hypoxic pulmonary hypertension: from cellular mechanisms to clinical phenotypes. Am J Physiol Lung Cell Mol Physiol 2015; 308: L229-L252. 
24 Shimoda LA, Semenza GL. HIF and the lung: role of hypoxia-inducible factors in pulmonary development and disease. Am J Respir Crit Care Med 2011; 183: 152-156.

25 Frid MG, Brunetti JA, Burke DL, et al. Hypoxia-induced pulmonary vascular remodeling requires recruitment of circulating mesenchymal precursors of a monocyte/macrophage lineage. Am J Pathol 2006; 168: 659-669.

26 Burke DL, Frid MG, Kunrath CL, et al. Sustained hypoxia promotes the development of a pulmonary artery-specific chronic inflammatory microenvironment. Am J Physiol Lung Cell Mol Physiol 2009; 297: L238-L250.

27 Kosanovic D, Dahal BK, Vroom C, et al. Selective inhibition of chemokine CCL2/MCP-1 reduces experimental pulmonary hypertension. Pneumologie 2013; 67: P10.

28 Ikeda Y, Yonemitsu Y, Kataoka C, et al. Anti-monocyte chemoattractant protein-1 gene therapy attenuates pulmonary hypertension in rats. Am J Physiol Heart Circ Physiol 2002; 283: H2021-H2028.

29 Yu YR, Mao L, Piantadosi CA, et al. CCR2 deficiency, dysregulation of Notch signaling, and spontaneous pulmonary arterial hypertension. Am J Respir Cell Mol Biol 2013; 48: 647-654.

30 Coppel J, Hennis P, Gilbert-Kawai E, et al. The physiological effects of hypobaric hypoxia versus normobaric hypoxia: a systematic review of crossover trials. Extrem Physiol Med 2015; 4: 2.

31 Mathew R. Cell-specific dual role of caveolin-1 in pulmonary hypertension. Pulm Med 2011; 2011: 573432.

32 Patel HH, Zhang S, Murray F, et al. Increased smooth muscle cell expression of caveolin-1 and caveolae contribute to the pathophysiology of idiopathic pulmonary arterial hypertension. FASEB J 2007; 21: 2970-2979.

33 Formenti F, Beer PA, Croft QP, et al. Cardiopulmonary function in two human disorders of the hypoxia-inducible factor (HIF) pathway: von Hippel-Lindau disease and HIF-2 $\alpha$ gain-of-function mutation. FASEB J 2011; 25 2001-2011.

34 Koeppen M, Lee JW, Seo SW, et al. Hypoxia-inducible factor 2- $\alpha$-dependent induction of amphiregulin dampens myocardial ischemia-reperfusion injury. Nat Commun 2018; 9: 816.

35 Smith TG, Brooks JT, Balanos GM, et al. Mutation of von Hippel-Lindau tumour suppressor and human cardiopulmonary physiology. PLoS Med 2006; 3: e290.

36 Gambaryan N, Perros F, Montani D, et al. Targeting of c-kit+ haematopoietic progenitor cells prevents hypoxic pulmonary hypertension. Eur Respir J 2011; 37: 1392-1399.

37 Vergadi E, Chang MS, Lee C, et al. Early macrophage recruitment and alternative activation are critical for the later development of hypoxia-induced pulmonary hypertension. Circulation 2011; 123: 1986-1995.

38 Tian W, Jiang X, Tamosiuniene R, et al. Blocking macrophage leukotriene B4 prevents endothelial injury and reverses pulmonary hypertension. Sci Transl Med 2013; 5: 200 ra117.

39 Pugliese SC, Kumar S, Janssen WJ, et al. A time- and compartment-specific activation of lung macrophages in hypoxic pulmonary hypertension. J Immunol 2017; 198: 4802-4812.

40 Dai Z, Zhu MM, Peng Y, et al. Therapeutic targeting of vascular remodeling and right heart failure in pulmonary arterial hypertension with a HIF-2 $\alpha$ inhibitor. Am J Respir Crit Care Med 2018; 198: 1423-1434.

41 Stenmark KR, Meyrick B, Galie N, et al. Animal models of pulmonary arterial hypertension: the hope for etiological discovery and pharmacological cure. Am J Physiol Lung Cell Mol Physiol 2009; 297: L1013-L1032.

42 Lythgoe MP, Rhodes CJ, Ghataorhe P, et al. Why drugs fail in clinical trials in pulmonary arterial hypertension, and strategies to succeed in the future. Pharmacol Ther 2016; 164: 195-203.

43 Wilkins MR. Apoptosis signal-regulating kinase 1 inhibition in pulmonary hypertension. too much to ASK? Am J Respir Crit Care Med 2018; 197: 286-288.

44 Li M, Riddle SR, Frid MG, et al. Emergence of fibroblasts with a proinflammatory epigenetically altered phenotype in severe hypoxic pulmonary hypertension. J Immunol 2011; 187: 2711-2722.

$45 \mathrm{Hu}$ CJ, Zhang $\mathrm{H}$, Laux A, et al. Mechanisms contributing to persistently activated cell phenotypes in pulmonary hypertension. J Physiol 2019; 597: 1103-1119. 